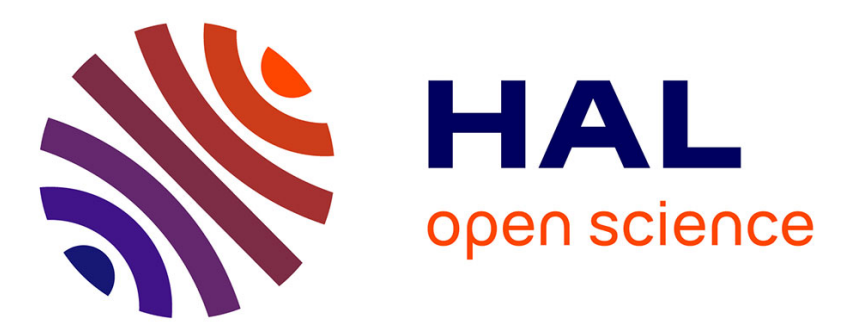

\title{
Lessons learned from WHISPER relaxation sounder in ten years of CLUSTER mission
}

Pierrette Décréau, Jean-Gabriel Trotignon, Patrick Canu, S. Kougblénou, G. Lointier, Jean-Louis Rauch, Xavier Vallières

\section{To cite this version:}

Pierrette Décréau, Jean-Gabriel Trotignon, Patrick Canu, S. Kougblénou, G. Lointier, et al.. Lessons learned from WHISPER relaxation sounder in ten years of CLUSTER mission. URSI 30Th Assembly, Aug 2011, Istambul, Turkey. 10.1109/URSIGASS.2011.6051119 . hal-02793732

\section{HAL Id: hal-02793732 \\ https://hal.science/hal-02793732}

Submitted on 8 Jul 2020

HAL is a multi-disciplinary open access archive for the deposit and dissemination of scientific research documents, whether they are published or not. The documents may come from teaching and research institutions in France or abroad, or from public or private research centers.
L'archive ouverte pluridisciplinaire HAL, est destinée au dépôt et à la diffusion de documents scientifiques de niveau recherche, publiés ou non, émanant des établissements d'enseignement et de recherche français ou étrangers, des laboratoires publics ou privés. 


\title{
Lessons learned from WHISPER relaxation sounder in ten years of CLUSTER mission
}

\author{
$\underline{\text { P. M. E. Décréau }}{ }^{1}$, J. -G. Trotignon ${ }^{1}$, P. Canu $^{2}$, S. Kougblenou ${ }^{1}$, G. Lointier ${ }^{1}$, J. -L. Rauch ${ }^{1}$ and \\ X. Vallières ${ }^{I}$
}

${ }^{1}$ Laboratoire de Physique et Chimie de l'Environnement et de l'Espace, 3A avenue de la recherche scientifique, 45071 Orléans, France; e-mail : pierrette.decreau@cnrs-orleans.fr, jean-gabriel.trotignon@cnrs-orleans.fr, sena.kougblenou@cnrs-orleans.fr, guillaume.lointier@cnrs-orleans.fr, jean-louis.rauch@cnrs-orleans.fr, xavier.vallieres@,cnrs-orleans.fr

${ }^{2}$ Laboratoire de Physique des Plasmas, Ecole Polytechnique, route de Saclay, 91128, Palaiseau, France; e-mail : patrick.canu@lpp.polytechnique.fr

\begin{abstract}
The four WHISPER instruments, part of the Wave Experiment Consortium on board the multi satellite CLUSTER mission, include each a relaxation sounder aimed to measure with a good precision and reliability the frequency positions of $F_{p}$, electron plasma frequency, and of $F_{c e}$, electron gyro-frequency. Those quantities give access to electron density and intensity of magnetic field, key parameters defining local plasma regime. WHISPER sounder's design and realization, based on the expertise provided by successful missions operated in late seventies and eighties (GEOS, ISEE, Viking), is characterized by use of a processor devoted to on board frequency analysis via FFT. This choice makes those relaxation sounders unique of their kind, allowing in particular a complete plasma diagnostic to be made in only $1.5 \mathrm{~s}$, almost one order of magnitude faster than in the past. The CLUSTER mission itself is unique, by its multi-point capability, and by its orbit, which along the mission allowed exploring a large variety of key regions of the magnetosphere. We present in this paper a few examples of WHISPER instrument behaviour and results, exploring in particular magnetosheath, polar cap, outer and inner plasmasphere. In the latter region, $F_{p}$ and $F_{c e}$ frequency values are far above the working frequency range of the instrument. This range, however, includes the lower hybrid frequency, $\mathrm{F}_{\mathrm{lh}}$, which shows up as a clear resonance triggered by the sounder, and allows measurement of $\mathrm{Ne}$ whenever $\mathrm{F}_{\mathrm{p}} / \mathrm{F}_{\mathrm{ce}}<1$.
\end{abstract}

\section{Introduction}

The CLUSTER mission is a multipoint observatory devoted to explore the magnetosphere. It needed four point measurements of plasma density, a key variable, with sufficient reliability and precision to estimate gradient vectors of this quantity. After the success of relaxation sounders on board missions like GEOS, ISEE, or Viking, it has been decided to embark such instruments on board each of the four CLUSTER satellites. The instrument built to this aim, called WHISPER (The Waves of HIgh frequency and Sounder for Probing of Electron density by Relaxation), combines two experiments to: (i) measure the natural emissions in the frequency band 2-80 kHz, and (ii) measure electron density and magnitude of magnetic field via a relaxation sounder [1]. The relaxation sounder allows a full plasma diagnostic performed in only $1.5 \mathrm{~s}$, almost one order of magnitude faster compared to earlier realizations. After 10 years in orbit, all four WHISPER instruments are functioning satisfactorily. In this paper, we present the instrument's design (section 2), how it behaves in various regions of magnetosphere encountered along an orbit (section 3). In section 4, an example of the recently observed low hybrid resonance near orbit perigee (now reaching altitudes $<2000 \mathrm{~km}$ ) is presented. Section 5 gives a short conclusion.

\section{WHISPER design}

In both facets of the WHISPER instrument (survey of natural waves, or active plasma diagnostic) the electric signal measured on one double sphere antenna is digitised and analysed in frequency in the $2-80 \mathrm{kHz}$ bandwidth. An on-board FFT processor delivers frequency spectra (power spectral density, amplitude only) at a resolution of 160 or $320 \mathrm{~Hz}$. In its active mode, the Whisper sounder triggers the medium to deliver an active spectrum where a number of resonances show up. Values of electron plasma frequency, $F_{p}$, and of $F_{c e}$, electron gyro-frequency, are determined after interpretation of the active spectrum transmitted on ground. WHISPER sounder operates as 
described in figure 1. It transmits a pulse (figure 1-a) formed by a sine wave train of short duration (1.0 or $0.5 \mathrm{~ms})$ centred at a frequency $f_{n}$. After a few milliseconds delay, the electric field present in the medium is recorded and analysed in frequency (figure 1-c). The frequency slice around $f_{n}(1 \mathrm{kHz}$ or $2 \mathrm{kHz})$ is copied as part of the active spectrum under construction. The next sine wave train is transmitted $27 \mathrm{~ms}$ later, at a frequency $\mathrm{f}_{\mathrm{n}+1}$, according to a table where $f_{n}$ and $f_{n+1}$ are separated of few $\mathrm{kHz}$ (figure 1-b) in order to avoid possible interferences between resonances triggered by two successive transmissions. The signal received is analysed and then the frequency slice around $\mathrm{f}_{\mathrm{n}+1}$ is copied as another brick of the active spectrum, and so on. The chosen frequency table covers, in its nominal version, the $4-80 \mathrm{kHz}$ range. It comprises a lower frequency range covered by $1.0 \mathrm{~ms}$ pulses, and a higher frequency range covered by $0.5 \mathrm{~ms}$ pulses, such that after 52 steps, the frequency range is totally covered. The complete process is performed in less than $1.5 \mathrm{~s}$, and repeated in order for the spinning antenna (at $\sim 4.0 \mathrm{~s}$ spin duration) to explore complementary orientations from one sweep to the other. After two successive sweeps, sounding operations give place to natural wave measurements.

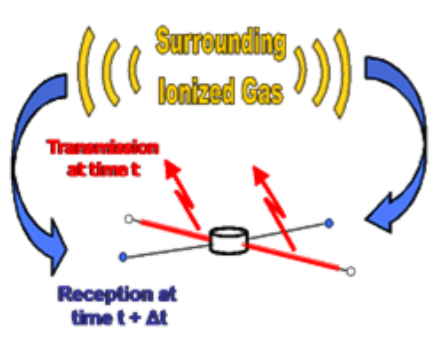

a) Transmission: sine wave-train $(\sim 1 \mathrm{~ms})$ of central frequency $f_{n}$

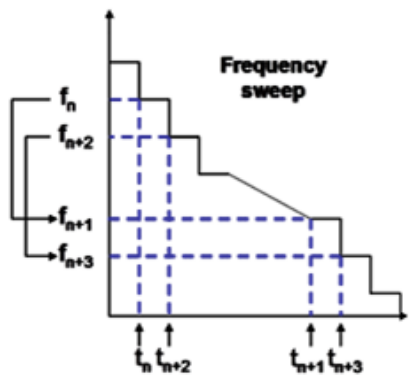

b) Frequency sweep: 80 frequency values from $4 \mathrm{kHz}$ to $82 \mathrm{kHz}$, covered by a non-linear time table $\mathrm{f}_{\mathrm{n}}\left(\mathrm{t}_{\mathrm{n}}\right)$

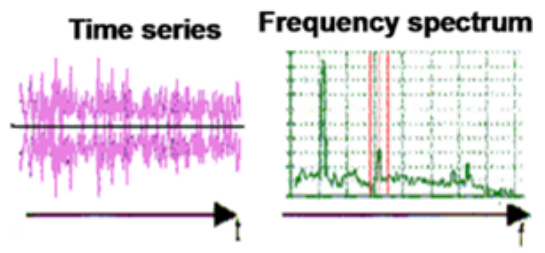

c) Reception \& wave analysis: A slice (centered on $f_{n}$ ) is selected after each sounding (6 freq. bins $-1 \mathrm{kHz}$ )

Fig. 1 Description of main sequences (1.5 s total duration) allowing constructing an active spectrum which, after interpretation on ground, provides one value of electron density.

A ground analysis of the resonance pattern present in the active spectrum allows identifying characteristic frequencies as $F_{p}$ and, in magnetized regions, $F_{c e}$ [2]. Typically, $3.0 \mathrm{~s}$ of sounding mode (two sweeps) are followed by $49 \mathrm{~s}$ of natural wave mode (delivering 11 frequency spectra transmitted to ground under nominal telemetry conditions). All four spacecraft follow the same time line (time tagged operations, at a common precision of a one second boundary clock). That strategy allows calculating the instantaneous space gradient vector of density, hence to inform about interesting spatial properties of the medium, independently of time variations.

\section{Observations in regions explored during the first mission phase}

Figure 2 displays frequency time spectrograms measured by the WHISPER instrument on CLUSTER spacecraft $\mathrm{C} 1$ on $9^{\text {th }}$ November, 2002, cumulating results obtained respectively during natural mode operations (medium panel) and during sounding operations (lower panel). Upper panel is obtained from OVT tool [3]. It shows Cluster orbits during this day (using standard colour code, black, red, green and purple, respectively for C1, C2, C3 and $\mathrm{C} 4$ ), and projected magnetic field lines for $\mathrm{C} 2$ positions (one hour apart), in Y-Z plane of GSM coordinate system.

Noting the different colour scales used respectively for the passive spectrogram (medium panel) and the active spectrogram (lower panel), some features are seen under both instrumental conditions, as the radio type III near 14:00 UT. Clear differences reflect the presence of resonances triggered by the sounder. In particular, the gyro frequency $F_{c e}$ and its harmonics show up when the satellite crosses the outer plasmasphere (from 00 to 02 UT), then the region over polar cap (from 02 to $08 \mathrm{UT}$ ). A single resonance at upper hybrid frequency $\mathrm{F}_{\mathrm{uh}}$ (such that $\mathrm{F}_{\mathrm{uh}}{ }^{2}=\mathrm{F}_{\mathrm{p}}{ }^{2}$ $+\mathrm{F}_{\mathrm{ce}}{ }^{2}$ ) is observed in addition, as well as, above $\mathrm{F}_{\mathrm{uh}}$, Bernstein mode resonances in each gyro-harmonic band. In the low latitude boundary layer (from 19 to $24 \mathrm{UT}$ ), a single resonance shows up, at $\mathrm{F}_{\mathrm{UH}}$ (very close to $\mathrm{F}_{\mathrm{p}}$ ). It is clear 
that, in several parts of the orbit, no reliable and accurate plasma diagnostic could have been made without sounding the medium, in particular in the polar cap and in the noisy low latitude boundary layer.
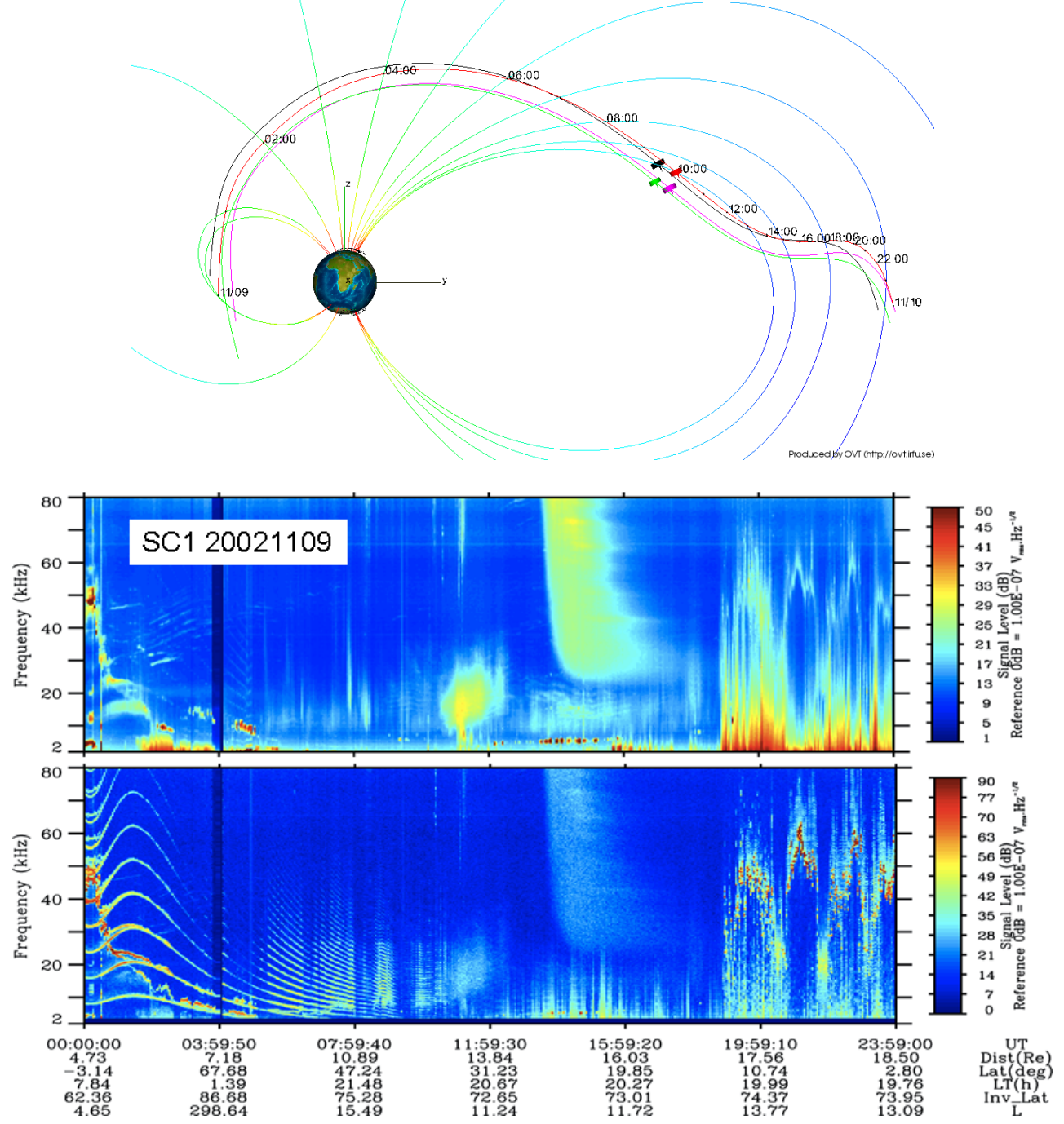

Fig. 2 CLUSTER orbits (top) and frequency time spectra measuring respectively natural waves (centre) and stimulated waves (bottom).

Figure 3 shows variations of density values during the 18:45 to 24:00 UT interval for the same day. At that position along orbit, $\mathrm{C} 1$ is the closest to Earth, measuring the lowest density. The gradient vector calculated along the orbit (not shown) is indeed opposite to Earth, directed toward magnetosheath.

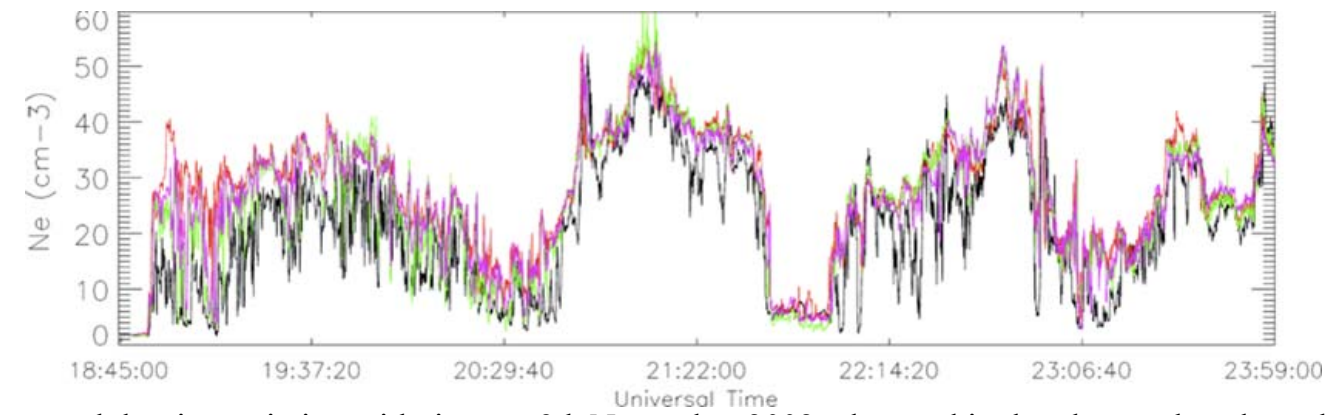

Fig. 3. Compared density variation with time on 9 th November 2002, observed in the plasma sheet boundary layer. 


\section{Triggering lower hybrid resonance at low altitude}

The lower hybrid frequency $\mathrm{F}_{\mathrm{lh}}$ reflects the coupling between ion and electron motions in a magnetized medium. WHISPER relaxation sounder triggers clear resonances at this frequency (figure 3 ). The $\mathrm{F}_{\mathrm{lh}}$ value depends of three variables: magnetic field amplitude, density and effective mass (related to the composition in term of fractional abundance of ions present in the medium), which vary with plasma regime encountered. The first one can be estimated from a model, or measured, with a good accuracy. A reasonable guess about effective mass can be made when $\mathrm{F}_{\mathrm{p}} / \mathrm{F}_{\mathrm{ce}}>1$ (in this case before 14:40 UT) and, when $\mathrm{F}_{\mathrm{p}} / \mathrm{F}_{\mathrm{ce}}<1$ (here after 14:45 UT), plasma density values can be derived from measured $\mathrm{F}_{\mathrm{lh}}$ frequencies [4].

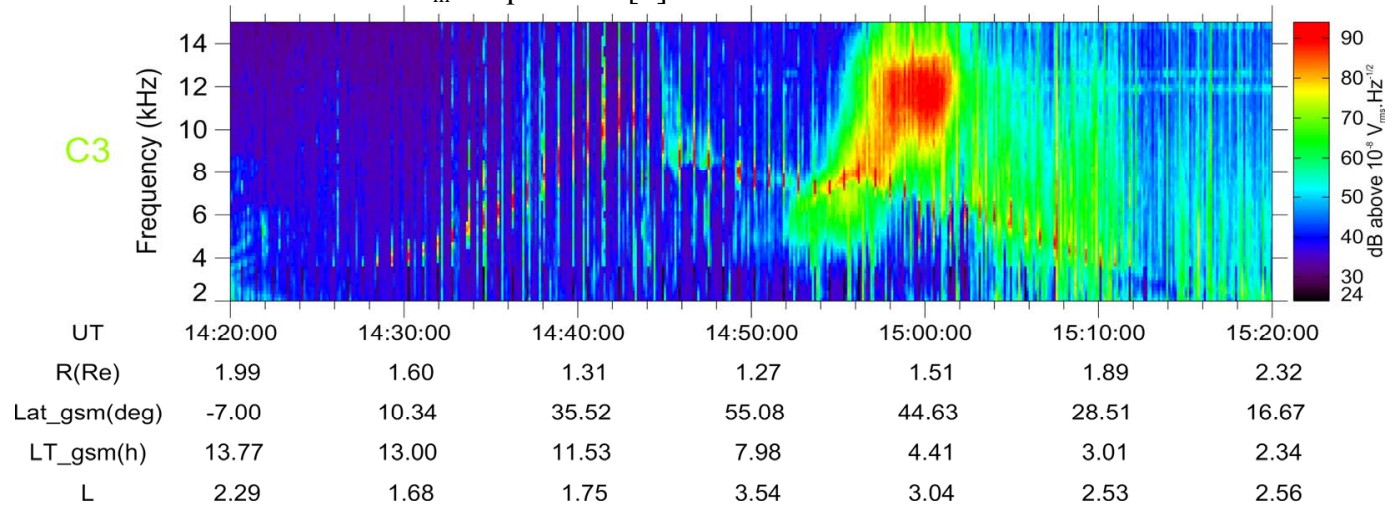

Fig. 4 Combined spectrogram measured recently $\left(31^{\text {st }}\right.$ October, 2010) at low altitudes by CLUSTER C3 satellite. Active spectra can be recognized by their low frequency limit $(4 \mathrm{kHz})$ contrasting with a lower limit $(2 \mathrm{kHz})$ for the passive (natural wave mode) spectra. $\mathrm{F}_{\mathrm{lh}}$ resonances show up in red colour.

\section{Conclusion}

WHISPER instrument has fulfilled its promises, allowing in addition to discover new capabilities of the relaxation sounder technique. A very rich database is archived in the CAA (CLUSTER ACTIVE ARCHIVE) built at ESTEC, ready for scientific investigations.

\section{Acknowledments}

Cluster and WHISPER instruments have been built thanks to CNES and ESA grants. Data analysis is supported by CNES and ESA.

\section{Bibliography}

[1] Décréau, P.M.E., P. Fergeau, V. Krasnosel'skikh, M. Lévêque, P. Martin, O. Randriamboarison, F.X. Sené, J.G. Trotignon, P. Canu, P.B. Mögensen and WHISPER Investigators, WHISPER, a resonance sounder and wave analyser : performances and perspectives for the CLUSTER misssion, Space Sci. Rev., 79, 157-193, 1997.

[2] Trotignon, J.-G., P.M.E. Décréau, J. L. Rauch, O. Randriamboarison, V. Krasnoselskikh, P. Canu, H. Alleyne, K. Yearby, E. Le Guirriec, H. C. Séran, F. X. Sené, Ph. Martin, M. Lévêque, P. Fergeau, How to determine the thermal electron density and the magnetic field strength from the CLUSTER/WHISPER observations around the Earth, Annales Geophysicae, 19, 1711-1720, 2001.

[3] http://ovt.irfu.se/

[4] Kougblenou, S., G. Lointier, P. M. E. Décréau, J. -G. Trotignon, J. -L. Rauch, X. Vallières, P. Canu, A. Masson, and J. Pickett, Lower hybrid resonances stimulated by the four relaxation sounders on board CLUSTER deep inside the plasmasphere: observations and inferred plasma characteristics, Cluster $10^{\text {th }}$ Anniversary workshop, 27 September - 1 October 2010, Corfu Island, Greece 